TRANSACTIONS OF THE

AMERICAN MATHEMATICAL SOCIETY

Volume 362, Number 12, December 2010, Pages 6381-6395

S 0002-9947(2010)04983-9

Article electronically published on July 15, 2010

\title{
TWO-DIMENSIONAL EULER FLOWS WITH CONCENTRATED VORTICITIES
}

\author{
MANUEL DEL PINO, PIERPAOLO ESPOSITO, AND MONICA MUSSO
}

\begin{abstract}
For a planar model of Euler flows proposed by Tur and Yanovsky (2004), we construct a family of velocity fields $\mathbf{w}_{\varepsilon}$ for a fluid in a bounded region $\Omega$, with concentrated vorticities $\omega_{\varepsilon}$ for $\varepsilon>0$ small. More precisely, given a positive integer $\alpha$ and a sufficiently small complex number $a$, we find a family of stream functions $\psi_{\varepsilon}$ which solve the Liouville equation with Dirac mass source,

$$
\Delta \psi_{\varepsilon}+\varepsilon^{2} e^{\psi_{\varepsilon}}=4 \pi \alpha \delta_{p_{a, \varepsilon}} \quad \text { in } \Omega, \quad \psi_{\varepsilon}=0 \quad \text { on } \partial \Omega
$$

for a suitable point $p=p_{a, \varepsilon} \in \Omega$. The vorticities $\omega_{\varepsilon}:=-\Delta \psi_{\varepsilon}$ concentrate in the sense that

$$
\omega_{\varepsilon}+4 \pi \alpha \delta_{p_{a, \varepsilon}}-8 \pi \sum_{j=1}^{\alpha+1} \delta_{p_{a, \varepsilon}+a_{j}} \rightarrow 0 \quad \text { as } \varepsilon \rightarrow 0,
$$

where the satellites $a_{1}, \ldots, a_{\alpha+1}$ denote the complex $(\alpha+1)$-roots of $a$. The point $p_{a, \varepsilon}$ lies close to a zero point of a vector field explicitly built upon derivatives of order $\leq \alpha+1$ of the regular part of Green's function of the domain.
\end{abstract}

\section{INTRODUCTION AND STATEMENT OF MAIN RESULTS}

We are concerned with stationary Euler equations for an incompressible and homogeneous fluid,

$$
\begin{cases}(\mathbf{w} \cdot \nabla) \mathbf{w}+\nabla p=0 & \text { in } \Omega, \\ \operatorname{div} \mathbf{w}=0 & \text { in } \Omega,\end{cases}
$$

where $\mathbf{w}$ is the velocity field and $p$ is the pressure. The domain $\Omega$ is either the whole $\mathbb{R}^{n}, n=2,3$, or a smooth, bounded domain $\Omega$. In the latter situation the velocity field $\mathbf{w}$ is naturally required to be tangent on $\partial \Omega$, that is,

$$
\mathbf{w} \cdot \nu=0 \quad \text { on } \partial \Omega
$$

$\nu$ being a unit normal vector to $\partial \Omega$. We shall restrict our investigation to the planar case $n=2$ when $\Omega$ is bounded and introduce the vorticity $\omega=$ curl $\mathbf{w}$. By applying

Received by the editors August 12, 2008.

2000 Mathematics Subject Classification. Primary 35J25, 35B25, 35B40.

Key words and phrases. $2 D$ Euler equations, singular Liouville equation, Liouville formula, concentrating solutions.

The first author was supported by grants Fondecyt 1070389 and Fondo Basal CMM.

The second author was supported by M.U.R.S.T., project "Variational methods and nonlinear differential equations".

The third author was supported by grants Fondecyt 1080099 and Anillo ACT 125 CAPDE.

(C)2010 American Mathematical Society Reverts to public domain 28 years from publication 
the curl operator to the first equation in (11), we are reduced to the Euler equations in vorticity form,

$$
\begin{cases}\mathbf{w} \cdot \nabla \omega=0 & \text { in } \Omega \\ \operatorname{div} \mathbf{w}=0 & \text { in } \Omega,\end{cases}
$$

supplemented by (2). We refer the reader to the books [25] and [23] for an exhaustive treatment of fluid mechanics models.

Let us further rewrite Problem (3). The second equation in (3) is equivalent to rewriting the velocity field $\mathbf{w}$ as

$$
\mathbf{w}=\left(\partial_{x_{2}} \psi,-\partial_{x_{1}} \psi\right) .
$$

In turn, the vorticity $\omega$ expresses as $\omega=-\Delta \psi$ in terms of $\psi$, the so-called stream function.

Now the ansatz $\omega=f(\psi)$ guarantees that the first equation in (3) is also automatically satisfied, and then the Euler equations reduce to solving the elliptic problem

$$
\Delta \psi+f(\psi)=0
$$

with Dirichlet boundary condition $\psi=0$ on $\partial \Omega$ on a bounded domain $\Omega$ to account for (2).

Many choices of $f$ are physically meaningful and lead to vortex-type configurations. The Stuart vortex pattern in [30] corresponds to $f(\psi)=\varepsilon^{2} e^{\psi}$. Tur and Yanovsky have recently proposed in 35] a singular ansatz

$$
f(\psi)=\varepsilon^{2} e^{\psi}-4 \pi \alpha \delta_{p}, \quad \alpha \in \mathbb{N},
$$

to describe vortex patterns of necklace type with $(\alpha+1)$-fold symmetry in rotational shear flow. Both papers [30, 35 consider Problem (4) in the whole $\mathbb{R}^{2}$ and explicit solutions are easily built according to Liouville's formula below. On a bounded domain $\Omega$, a statistical mechanics approach in [5, 6, 20, has provided a rigorous derivation of Stuart's ansatz.

In this paper we consider the Tur-Yanovsky problem on a bounded domain $\Omega$, a much harder issue to pursue. In terms of the stream function $\psi$ we are thus led to the singular Liouville equation

$$
\left\{\begin{array}{cl}
\Delta \psi+\varepsilon^{2} e^{\psi}=4 \pi \alpha \delta_{p} & \text { in } \Omega, \\
\psi=0 & \text { on } \partial \Omega .
\end{array}\right.
$$

The parameter $\varepsilon>0$ is small and, as we will see, its smallness will yield to flows having vorticities $\omega$ concentrated on small "blobs".

Liouville-type equations arise also in several superconductivity theories in the self-dual regime, as for the abelian Maxwell-Higgs and Chern-Simons-Higgs theories. In the latter model, a mean field form of Problem (5) on the torus arises as a limiting equation for nontopological condensates as the Chern-Simons parameter tends to zero as shown in [28, 32]. Problem (55) is a limiting model equation in this context and explains why it has attracted a lot of attention, as we describe precisely below.

In a superconducting sample $\Omega$ a Dirichlet boundary condition $\varphi$ can be imposed and the homogeneous case $\varphi=0$, discussed in [18, is especially interesting since it describes a perfectly superconductive regime on $\partial \Omega$. 
The regular case $\alpha=0$ in Problem (5), sometimes referred to as the Gelfand problem [16,

$$
\left\{\begin{array}{cl}
\Delta \psi+\varepsilon^{2} e^{\psi}=0 & \text { in } \Omega, \\
\psi=0 & \text { on } \partial \Omega,
\end{array}\right.
$$

has been broadly considered in the literature. When $\varepsilon>0$ is sufficiently small, the existence of both a small and a large solution as $\varepsilon \rightarrow 0$ has long been known as first found in [11, 19]. In the language of the calculus of variations, applied to the functional

$$
J(\psi)=\frac{1}{2} \int_{\Omega}|\nabla \psi|^{2}-\varepsilon^{2} \int_{\Omega} e^{\psi}, \quad \psi \in H_{0}^{1}(\Omega),
$$

the small solution corresponds to a local minimizer, while the large solution is a mountain pass critical point. The blowing-up behavior of a large solution was first described in [36] when $\Omega$ is simply connected. In the general case, the analysis in the works $4,22,26,27,31$ yields that, if $\psi_{\varepsilon}$ is a family of blowing-up solutions of (6) with $\varepsilon^{2} \int_{\Omega} e^{\psi_{\varepsilon}}$ uniformly bounded, then, up to subsequences, there is an integer $k \geq 1$ such that $\varepsilon^{2} \int_{\Omega} e^{\psi_{\varepsilon}} \rightarrow 8 \pi k$. Moreover, there are points $\xi_{1}^{\varepsilon}, \ldots, \xi_{k}^{\varepsilon}$ in $\Omega$, which remain away one from each other and away from $\partial \Omega$, such that

$$
\varepsilon^{2} e^{\psi_{\varepsilon}}-\sum_{i=1}^{k} 8 \pi \delta_{\xi_{i}^{\varepsilon}} \rightarrow 0 \quad \text { as } \varepsilon \rightarrow 0
$$

in the measure sense. Besides,

$$
\nabla \varphi_{k}\left(\xi_{1}^{\varepsilon}, \ldots, \xi_{k}^{\varepsilon}\right) \rightarrow 0 \quad \text { as } \varepsilon \rightarrow 0
$$

where

$$
\varphi_{k}\left(\xi_{1}, \ldots, \xi_{k}\right):=\left\{\begin{array}{cc}
H\left(\xi_{1}, \xi_{1}\right) & \text { if } k=1, \\
\sum_{i=1}^{k} H\left(\xi_{i}, \xi_{i}\right)+\sum_{i \neq j} G\left(\xi_{i}, \xi_{j}\right) & \text { if } k \neq 1 .
\end{array}\right.
$$

Here $G(z, \xi)$ denotes the Dirichlet Green's function in $\Omega$, namely

$$
G(z, \xi)=\Gamma(z-\xi)+H(z, \xi), \quad \Gamma(z):=\frac{1}{2 \pi} \log \frac{1}{|z|},
$$

where $H(z, \xi)$, its regular part, satisfies

$$
\begin{cases}\Delta_{z} H(\cdot, \xi)=0 & \text { in } \Omega, \\ H(\cdot, \xi)=-\Gamma(\cdot-\xi) & \text { on } \partial \Omega .\end{cases}
$$

Moreover, refined asymptotic estimates hold, as established in a general setting in [7, 21, which lead to the computation of the Leray-Schauder degree of an associated nonlinear operator in [8]. In particular, $\psi_{\varepsilon}$ satisfies

$$
\psi_{\varepsilon}(z)=8 \pi \sum_{i=1}^{k} G\left(z, \xi_{i}^{\varepsilon}\right)+o(1), \quad \psi_{\varepsilon}(z)=\log \frac{8 \mu_{i}^{2}}{\left(\mu_{i}^{2} \varepsilon^{2}+\left|z-\xi_{i}^{\varepsilon}\right|^{2}\right)^{2}}+o(1),
$$

respectively, away from all points $\xi_{i}^{\varepsilon}$, and around each of them, for some $\mu_{i}>0$. The family of functions

$$
w_{\varepsilon, \mu, \xi}(z):=\log \frac{8 \mu^{2}}{\left(\mu^{2} \varepsilon^{2}+|z-\xi|^{2}\right)^{2}}
$$


corresponds to all solutions of the equation

$$
\Delta w+\varepsilon^{2} e^{w}=0 \text { in } \mathbb{R}^{2},
$$

such that $\int_{\mathbb{R}^{2}} e^{w}<+\infty$; see 9 . Besides,

$$
\varepsilon^{2} \int_{\mathbb{R}^{2}} e^{w_{\varepsilon, \mu, \xi}}=8 \pi, \quad \varepsilon^{2} e^{w_{\varepsilon, \mu, \xi}} \rightarrow 8 \pi \delta_{\xi} \quad \text { as } \varepsilon \rightarrow 0 .
$$

The reciprocal issue, namely the existence of solutions with the above properties, has been addressed in 11, near nondegenerate critical points of $\varphi_{k}$, and in [12, 15] associated to topologically nontrivial critical point situations for $\varphi_{k}$. In particular, it is found in [12] that $\psi_{\varepsilon}$ as above exists for any $k \geq 1$ provided that the domain is not simply connected.

Important progress in the understanding of blowing-up solutions of Problem (5) with $\alpha>0$ has been achieved in the local analysis in the works 2, 3, 34 from the point of view of quantization of blow-up levels and Harnack-type estimates. See 33. for a complete account on the topic.

Concerning construction of solutions to (5) with $\alpha>0$, only a few results are available. The first important remark is that the functions

$$
w_{\varepsilon, \mu}(z)=-4 \pi \alpha \Gamma(z-p)+\log \frac{8 \mu^{2}(1+\alpha)^{2}}{\left(\mu^{2} \varepsilon^{2}+|z-p|^{2+2 \alpha}\right)^{2}}
$$

satisfy precisely

$\Delta w+\varepsilon^{2} e^{w}=4 \pi \alpha \delta_{p}, \quad \varepsilon^{2} \int_{\mathbb{R}^{2}} e^{w_{\varepsilon, \mu}}=8 \pi(1+\alpha), \quad \varepsilon^{2} e^{w_{\varepsilon, \mu}} \rightarrow 8 \pi(1+\alpha) \delta_{p}$ as $\varepsilon \rightarrow 0$.

When $\alpha \in \mathbb{N}$, an integer, the family above extends to one carrying an extra parameter $a$ which plays a similar role as $\xi$ in (11): in complex notation, all functions

$$
w_{\varepsilon, \mu, a}(z)=-4 \pi \alpha \Gamma(z-p)+\log \frac{8 \mu^{2}(1+\alpha)^{2}}{\left(\mu^{2} \varepsilon^{2}+\left|(z-p)^{\alpha+1}-a\right|^{2}\right)^{2}}
$$

satisfy (13) with the third property replaced by

$$
\varepsilon^{2} e^{w_{\varepsilon, \mu, a}} \rightarrow 8 \pi \sum_{j=1}^{\alpha+1} \delta_{p+a_{j}} \quad \text { as } \varepsilon \rightarrow 0,
$$

where the $a_{j}$ 's are the complex $(\alpha+1)$-roots of $a$.

The difference between the cases $\alpha \in \mathbb{N}$ and $\alpha \notin \mathbb{N}$ is not just cosmetics but analytically essential. In the latter case, a suitable form of nondegeneracy up to dilations holds for the solutions (12), which allows in 13, 14, the construction of solutions to (5) so that away from $p$,

$$
\psi_{\varepsilon}(z)=-4 \pi \alpha G(z, p)+8 \pi(1+\alpha) G(z, p)+o(1) \text { and } \varepsilon^{2} e^{\psi_{\varepsilon}} \rightarrow 8 \pi(1+\alpha) \delta_{p} \text { as } \varepsilon \rightarrow 0 \text {. }
$$

On the other hand, solutions with concentration points away from $p$ have been built in [12, regardless whether or not $\alpha$ is an integer. Whenever $k<1+\alpha$, there is a solution $\psi_{\varepsilon}$ of (5) and, up to a subsequence, $k$ points $\xi_{j}^{\varepsilon} \in \Omega \backslash\{p\}$ such that away 
from them,

$\psi_{\varepsilon}(z)=-4 \pi \alpha G(z, p)+8 \pi \sum_{j=1}^{k} G\left(z, \xi_{j}^{\varepsilon}\right)+o(1)$ and $\varepsilon^{2} e^{\psi_{\varepsilon}}-8 \pi \sum_{j=1}^{k} \delta_{\xi_{j}^{\varepsilon}} \rightarrow 0$ as $\varepsilon \rightarrow 0$.

A natural, unsettled question for which the methods in 12, 14 fail is whether in case $\alpha \in \mathbb{N}$, solutions $\psi_{\varepsilon}$ with property (16), or for $k=\alpha+1$ with property (17) exist. Expressions (14) and (15) suggest that both scenarios may be possible, using these functions, suitably corrected, as approximations of a solution of (5).

The case $\alpha \in \mathbb{N}$ in Problem (5) is more difficult, and at the same time the most relevant to physical applications. Our main result states that both of the above situations do take place; however, the location of the vortex $p$ does not seem possible to be prescribed in an arbitrary way as in [13, 14] in case $\alpha \notin \mathbb{N}$ but rather, as in the situation of standard type II superconductivity, it is determined by the geometry of the region and boundary conditions in an $\varepsilon$-dependent way.

Let us assume that $\Omega$ is simply connected and consider a holomorphic extension

$$
h_{p}(z)=H(z, p)-H(p, p)+i \tilde{H}(z, p) \text { in } \Omega
$$

of the harmonic function $H(z, p)-H(p, p)$ so that $h_{p}(p)=0$. This means $\tilde{H}(p, p)=$ 0 and

$$
\tilde{H}_{z_{1}}(z, p)=H_{z_{2}}(z, p), \quad \tilde{H}_{z_{2}}(z, p)=-H_{z_{1}}(z, p) \text { for all } z \in \Omega .
$$

Then we let

$$
\Psi(p)=\frac{d^{\alpha+1}}{d z^{\alpha+1}}\left(e^{2 \pi(\alpha+2) h_{p}(z)}\right)(p) .
$$

Our main result for problem (5) is stated as follows.

Theorem 1.1. Assume that $\alpha \in \mathbb{N}$ and that $\Omega$ is simply connected. Then there exists $\delta>0$ such that for each a with $|a| \leq \delta$, there is a point $p_{a, \varepsilon} \in \Omega$ away from $\partial \Omega$ and a flow described by $\psi_{\varepsilon}$, the solution of

$$
\left\{\begin{array}{cl}
\Delta \psi_{\varepsilon}+\varepsilon^{2} e^{\psi_{\varepsilon}}=4 \pi \alpha \delta_{p_{a \varepsilon}} & \text { in } \Omega, \\
\psi_{\varepsilon}=0 & \text { on } \partial \Omega,
\end{array}\right.
$$

for which the associated vorticity $\omega_{\varepsilon}:=\varepsilon^{2} e^{\psi_{\varepsilon}}-4 \pi \alpha \delta_{p_{a, \varepsilon}}$ is concentrated in the sense that

$$
\omega_{\varepsilon}+4 \pi \alpha \delta_{p_{a, \varepsilon}}-8 \pi \sum_{j=1}^{\alpha+1} \delta_{p_{a, \varepsilon}+a_{j}} \rightarrow 0 \quad \text { as } \varepsilon \rightarrow 0
$$

where the $a_{j}$ 's are the complex $(\alpha+1)$-roots of a. Besides we have

$$
\Psi\left(p_{a, \varepsilon}\right) \rightarrow 0 \quad \text { as }|a|+\varepsilon \rightarrow 0 .
$$

For $\alpha \geq 1$, we observe that $\alpha+1$ vertices of any sufficiently tiny regular polygon can be allocated with a suitable center to yield these vertices as an asymptotic concentration set. The solution predicted satisfies

$$
\psi_{\varepsilon}(z)=-4 \pi \alpha G\left(z, p_{a, \varepsilon}\right)+8 \pi \sum_{j=1}^{\alpha+1} G\left(z, p_{a, \varepsilon}+a_{j}\right)+o(1) \quad \text { as } \varepsilon \rightarrow 0
$$

away from the concentration points. Let us observe that this result recovers the one known for $\alpha=0$, where $p_{a, \varepsilon}=\xi^{\varepsilon}-a$ and where $\xi^{\varepsilon}$, the point of concentration, 
approaches a critical point of the Robin's function. Let us notice that indeed, for $\alpha=0$

$$
\Psi(p)=0 \Longleftrightarrow \nabla_{z} H(p, p)=0 .
$$

In general, the condition $\Psi(p)=0$ involves derivatives of orders up to $\alpha+1$ of the function $H(z, p)$ at $z=p$. Notice also that the function $\Psi(p)$ is well-defined even if $\Omega$ is not simply connected. We suspect that this requirement may be lifted but our proof uses this fact. Theorem 1.1 will be a consequence of a more general result, which states that, associated to any region $\Lambda \subset \Omega$ for which $\operatorname{deg}(\Psi, \Lambda, 0) \neq 0$, a solution as in Theorem 1.1 exists with concentration points inside $\Lambda$. The proof applies with just slight changes to the case of nonzero boundary data $\varphi$.

\section{A more General Result}

In what follows we assume that $\Omega$ is a simply connected bounded domain. Theorem 1.1 will be a consequence of a more general result concerning the Dirichlet problem

$$
\left\{\begin{array}{cl}
\Delta u+\varepsilon^{2} e^{u}=4 \pi N \delta_{p} & \text { in } \Omega, \\
u=\varphi & \text { on } \partial \Omega,
\end{array}\right.
$$

where $\varphi$ is a smooth function and $N \geq 1$ is an integer. Let $\Phi$ be the harmonic extension of $\varphi$ to all of $\Omega$. The substitution

$$
v(z):=u(z)+4 \pi N G(z, p)-\Phi(z)
$$

transforms Problem (19) into the (regular) boundary value problem

$$
\left\{\begin{array}{cl}
\Delta v+\varepsilon^{2}|z-p|^{2 N} e^{2 K(z)} e^{v}=0 & \text { in } \Omega, \\
v=0 & \text { on } \partial \Omega
\end{array}\right.
$$

where

$$
2 K(z)=\Phi(z)-4 \pi N H(z, p) .
$$

The homogeneous case $\varphi=0$ corresponds to simply having $K(z)=-2 \pi N H(z, p)$.

In what follows, we identify $\left(z_{1}, z_{2}\right) \in \mathbb{R}^{2}$ and $z=z_{1}+i z_{2} \in \mathbb{C}$. We can associate to (20) a limiting problem of Liouville type, which will play a crucial role in the construction of solutions blowing up at $p$ as $\varepsilon \rightarrow 0^{+}$:

$$
\left\{\begin{array}{l}
\Delta V+\varepsilon^{2}|z-p|^{2 N} e^{V}=0 \text { in } \mathbb{R}^{2} \\
\int_{\mathbb{R}^{2}}|z-p|^{2 N} e^{V}<\infty
\end{array}\right.
$$

Let us recall Liouville's formula 24]. Given a holomorphic function $f$ on $\mathbb{C}$, the function

$$
\ln \frac{8\left|f^{\prime}(z)\right|^{2}}{\left(1+|f(z)|^{2}\right)^{2}}-2 \ln \varepsilon
$$

solves the equation $\Delta V+\varepsilon^{2} e^{V}=0$ in the set $\left\{z \in \mathbb{C} \mid f^{\prime}(z) \neq 0\right\}$. We can allow $f(z)$ to have simple poles since the Liouville formula (22) still makes sense.

If now $f^{\prime}$ vanishes only at the point $p$ with multiplicity $N$, the function

$$
\ln \frac{8\left|f^{\prime}(z)\right|^{2}}{\left(1+|f(z)|^{2}\right)^{2}}-2 \ln \varepsilon-\ln |z-p|^{2 N}
$$


solves the equation in (21) but possibly does not have energy $\int_{\mathbb{R}^{2}}|z-p|^{2 N} e^{V}$ finite. The finite energy condition in the entire space forces the choice

$$
f(z)=\frac{(z-p)^{N+1}-a}{\mu \varepsilon}, \quad \mu>0, a \in \mathbb{C},
$$

which leads exactly to the following three-parameter family of solutions to (21):

$$
\tilde{V}_{a}(z)=\ln \frac{8(N+1)^{2} \mu^{2}}{\left(\mu^{2} \varepsilon^{2}+\left|(z-p)^{N+1}-a\right|^{2}\right)^{2}}, \quad z \in \mathbb{C} ;
$$

see [10, 29]. Let us note that $\tilde{V}_{a}(z)$ is the regular part of $w_{\varepsilon, \mu, a}$ as defined in (14). For $a=|a| e^{i \theta} \in \mathbb{C}$, we consider the $(N+1)$-roots of $a$,

$$
a_{j}=|a|^{\frac{1}{N+1}} e^{i \frac{\theta}{N+1}+\frac{2 \pi i}{N+1}(j-1)}, \quad j=1, \ldots, N+1,
$$

where $i$ is the imaginary unit in $\mathbb{C}$.

The function $\tilde{V}_{a}(z)$ solves the PDE in (20) with $K=0$ but does not have the right boundary condition. By the Liouville formula, we will choose more carefully the function $f(z)$ to achieve the Dirichlet boundary condition and include the potential $e^{2 K(z)}$ in the equation. Our approach is related to that by Weston in [36] for $N=0$. Nonetheless, the assumptions in 36 , on the Riemann conformal map of $\Omega$ onto the unit disc are shown here to be unnecessary, and our improved approach is also essential to deal with a nonzero $N \in \mathbb{N}$.

We explain below how to choose $f(z)$, and the simply connectedness of $\Omega$ will be crucial. The details of our construction will be presented in Section 3 .

Let $Q \in C(\bar{\Omega}, \mathbb{C})$ be a holomorphic function in $\Omega$ so that

$$
Q(p)=1, \quad Q(z)-\frac{z-p}{N+1} Q^{\prime}(z) \neq 0 \quad \text { in } \Omega .
$$

Since, as already observed, $f$ can have simple poles, let us take

$$
f(z)=\frac{(z-p)^{N+1} Q^{-1}(z)-a}{\mu \varepsilon}
$$

in Liouville's formula (23) in order to get a solution

$$
V_{a}(z)=\ln \frac{8(N+1)^{2} \mu^{2}\left|Q(z)-\frac{z-p}{N+1} Q^{\prime}(z)\right|^{2}}{\left(\mu^{2} \varepsilon^{2}|Q(z)|^{2}+\left|(z-p)^{N+1}-a Q(z)\right|^{2}\right)^{2}}
$$

of the equation

$$
-\Delta v=\varepsilon^{2}|z-p|^{2 N} e^{v} \quad \text { in } \Omega .
$$

Given the projection operator $P: H^{1}(\Omega) \rightarrow H_{0}^{1}(\Omega)$, we will search for $Q$ and $\mu$ (depending on $a$ and $\varepsilon>0$ small) such that $P V_{a}=V_{a}-2 K$. The function $P V_{a}$ will then be a solution of (20).

Since $Q-\frac{z-p}{N+1} Q^{\prime} \neq 0$ in $\Omega$, the harmonic function

$R_{\mu, Q, a}(z)=\frac{1}{2}\left[P V_{a}-V_{a}+\ln \left(8(N+1)^{2} \mu^{2}\right)+\ln \left|Q(z)-\frac{z-p}{N+1} Q^{\prime}(z)\right|^{2}-8 \pi H_{Q, a}(z)\right]$

satisfies by the Maximum Principle $R_{\mu, Q, a}=O\left(\varepsilon^{2}\right)$ uniformly in $\Omega$, where $H_{Q, a}(z)$ is the solution of

$$
\begin{cases}\Delta H=0 & \text { in } \Omega \\ H(z)=\frac{1}{2 \pi} \ln \left|(z-p)^{N+1}-a Q(z)\right| & \text { on } \partial \Omega .\end{cases}
$$


Observe that

$$
H_{Q, 0}(z)=(N+1) H(z, p),
$$

where $H(z, p)$ is the regular part of the Green's function at $p$. Since $\Omega$ is a simply connected domain, let $h_{Q, a}(z), r_{\mu, Q, a}(z)$ and $k(z)$ be the holomorphic extensions in $\Omega$ of the harmonic functions $H_{Q, a}(z)-H_{Q, a}(p), R_{\mu, Q, a}(z)-R_{\mu, Q, a}(p)$ and $K(z)-$ $K(p)$, respectively, so that $h_{Q, a}(p)=r_{\mu, Q, a}(p)=k(p)=0$. Set

$$
c_{\mu, Q, a}=\frac{1}{(N+1) !} \frac{d^{N+1}}{d z^{N+1}}\left(e^{4 \pi h_{Q, a}(z)+r_{\mu, Q, a}(z)+k(z)}\right)(p) .
$$

For $a$ and $\varepsilon>0$ small, in Section 3 we will find a solution $Q_{\mu, a}$ of the equation:

$$
\begin{aligned}
& -\ln \left|Q(z)-\frac{z-p}{N+1} Q^{\prime}(z)\right|+4 \pi H_{Q, a}(z)+R_{\mu, Q, a}(z)+K(z) \\
& =4 \pi H_{Q, a}(p)+R_{\mu, Q, a}(p)+K(p)+\operatorname{Re}\left(c_{\mu, Q, a}(z-p)^{N+1}\right)
\end{aligned}
$$

in $\Omega$, where Re stands for the real part of a complex number. Next, we will solve

$$
\mu=\left.\frac{e^{4 \pi H_{Q, a}(p)+R_{\mu, Q, a}(p)+K(p)}}{\sqrt{8}(N+1)}\right|_{Q=Q_{\mu, a}}
$$

for some $\mu_{a}$, where $a$ and $\varepsilon>0$ are small parameters. Setting $Q_{a}=Q_{\mu_{a}, a}$, the function

satisfies:

$$
V_{a}(z)=\ln \frac{8(N+1)^{2} \mu_{a}^{2}\left|Q_{a}(z)-\frac{z-p}{N+1} Q_{a}^{\prime}(z)\right|^{2}}{\left(\mu_{a}^{2} \varepsilon^{2}\left|Q_{a}(z)\right|^{2}+\left|(z-p)^{N+1}-a Q_{a}(z)\right|^{2}\right)^{2}}
$$

$$
P V_{a}=V_{a}-2 K(z)+2 \operatorname{Re}\left(c_{a}(z-p)^{N+1}\right) \quad \text { in } \Omega,
$$

where $c_{a}:=c_{\mu_{a}, Q_{a}, a}$.

As a conclusion, by means of (27), Problem (20) reduces to solving the equation

$$
c_{a}(p)=\frac{1}{(N+1) !} \frac{d^{N+1}}{d z^{N+1}}\left(e^{4 \pi h_{Q_{a}, a}(z)+r_{\mu_{a}, Q_{a}, a}(z)+k(z)}\right)(p)=0 .
$$

This implies that every "stable" isolated zero point $p_{0} \in \Omega$ for the map

$$
\Psi(p):=\frac{d^{N+1}}{d z^{N+1}}\left(e^{4 \pi(N+1) h_{p}(z)+k(z)}\right)(p),
$$

in the sense that $\Psi$ has a nonzero local index at $p_{0}$, will provide us with points $p_{a, \varepsilon}$, for $a$ and $\varepsilon>0$ small, so that $c_{a}=0$. More generally, we have the validity of the following result:

Theorem 2.1. Let $\Lambda \Subset \Omega$ be a region such that $\operatorname{deg}(\Psi, \Lambda, 0) \neq 0$. Then there exists $\delta>0$ such that for each a with $|a| \leq \delta$, there is a point $p_{a, \varepsilon} \in \Lambda$ so that Problem (20) for $p=p_{a, \varepsilon}$ has a solution $v_{\varepsilon}$ with

$$
\varepsilon^{2}\left|z-p_{a, \varepsilon}\right|^{2 N} e^{2 K(z)} e^{v_{\varepsilon}}-8 \pi \sum_{j=1}^{N+1} \delta_{p_{a, \varepsilon}+a_{j}} \rightarrow 0 \quad \text { as } \varepsilon \rightarrow 0,
$$

where the $a_{j}$ 's are the complex $(N+1)$-roots of a. Besides we have

$$
\Psi\left(p_{a, \varepsilon}\right) \rightarrow 0 \text { as }|a|+\varepsilon \rightarrow 0 .
$$

Let us stress that if $p_{0}$ is a stable isolated zero of $\Psi$, then we can obtain solutions as above with $p_{a, \varepsilon} \rightarrow p_{0}$ as $|a|+\varepsilon \rightarrow 0$.

In what remains of this paper we will prove Theorem 2.1 with the scheme described above and deduce Theorem 1.1 as a corollary. 


\section{The Reduction to $c_{a}=0$}

Let $Q \in C(\bar{\Omega}, \mathbb{C})$ be a holomorphic function in $\Omega$ so that $Q(p)=1$ and

$$
Q(z)-\frac{z-p}{N+1} Q^{\prime}(z) \neq 0 \quad \text { for all } z \in \Omega
$$

Let

$$
V_{a}(z)=\ln \frac{8(N+1)^{2} \mu^{2}\left|Q(z)-\frac{z-p}{N+1} Q^{\prime}(z)\right|^{2}}{\left(\mu^{2} \varepsilon^{2}|Q(z)|^{2}+\left|(z-p)^{N+1}-a Q(z)\right|^{2}\right)^{2}},
$$

and denote by $P V_{a}$ the projection of $V_{a}$ onto the space $H_{0}^{1}(\Omega)$. Namely $P V_{a}$ is the unique solution of

$$
\begin{cases}-\Delta P V_{a}=-\Delta V_{a}=\varepsilon^{2}|z-p|^{2 N} e^{V_{a}} & \text { in } \Omega \\ P V_{a}=0 & \text { on } \partial \Omega .\end{cases}
$$

For $a$ small, let $H_{Q, a}(z)$ be the solution of

$$
\begin{cases}\Delta H=0 & \text { in } \Omega, \\ H(z)=\frac{1}{2 \pi} \ln \left|(z-p)^{N+1}-a Q(z)\right| & \text { on } \partial \Omega .\end{cases}
$$

Since we assume $Q-\frac{z-p}{N+1} Q^{\prime} \neq 0$ in $\Omega$, the function $\ln \left|Q(z)-\frac{z-p}{N+1} Q^{\prime}(z)\right|$ is harmonic in $\Omega$. Since the harmonic function

$$
\begin{gathered}
R_{\mu, Q, a}(z)=\frac{1}{2}[ \\
{\left[V_{a}(z)-V_{a}(z)+\ln \left(8(N+1)^{2} \mu^{2}\right)+\ln \mid Q(z)\right.} \\
\left.-\left.\frac{z-p}{N+1} Q^{\prime}(z)\right|^{2}-8 \pi H_{Q, a}(z)\right]
\end{gathered}
$$

satisfies $R_{\mu, Q, a}=O\left(\varepsilon^{2}\right)$ uniformly on $\partial \Omega$ as $\varepsilon \rightarrow 0$ (together with any boundary derivatives), by elliptic estimates (see [17]) we get that

$$
\begin{array}{r}
P V_{a}=V_{a}-\ln \left(8(N+1)^{2} \mu^{2}\right)-\ln \left|Q(z)-\frac{z-p}{N+1} Q^{\prime}(z)\right|^{2}+8 \pi H_{Q, a}(z)+2 R_{\mu, Q, a}(z), \\
R_{\mu, Q, a}=O\left(\varepsilon^{2}\right)
\end{array}
$$

in $C^{1}(\bar{\Omega})$. Let $h_{Q, a}(z), r_{\mu, Q, a}(z)$ and $k(z)$ be the holomorphic extensions in $\Omega$ of the harmonic functions $H_{Q, a}(z)-H_{Q, a}(p), R_{\mu, Q, a}(z)-R_{\mu, Q, a}(p)$ and $K(z)-K(p)$ so that $h_{Q, a}(p)=r_{\mu, Q, a}(p)=k(p)=0$. Set

$$
c_{\mu, Q, a}=\frac{1}{(N+1) !} \frac{d^{N+1}}{d z^{N+1}}\left(e^{4 \pi h_{Q, a}(z)+r_{\mu, Q, a}(z)+k(z)}\right)(p) .
$$

It suffices for equation (25) to hold true that $Q(z)$ satisfies the equation

$$
Q(z)-\frac{z-p}{N+1} Q^{\prime}(z)=\exp \left(4 \pi h_{Q, a}(z)+r_{\mu, Q, a}(z)+k(z)-c_{\mu, Q, a}(z-p)^{N+1}\right),
$$

or equivalently

$$
\begin{aligned}
& Q(z)=-(N+1)(z-p)^{N+1} \int \frac{d z}{(z-p)^{N+2}} \exp ( 4 \pi h_{Q, a}(z)+r_{\mu, Q, a}(z) \\
&\left.+k(z)-c_{\mu, Q, a}(z-p)^{N+1}\right),
\end{aligned}
$$


where the symbol $\int$ designates a primitive in $\Omega$ of the argument function. The choice of the complex constant $c=c_{\mu, Q, a}$ guarantees that the right hand side in expression (32) is a well-defined single-valued function. Indeed, let us set

$$
W(z):=\exp \left(4 \pi h_{Q, a}(z)+r_{\mu, Q, a}(z)+k(z)\right)
$$

and

$$
\begin{aligned}
\Sigma(z) & :=\exp \left(4 \pi h_{Q, a}(z)+r_{\mu, Q, a}(z)+k(z)-c(z-p)^{N+1}\right) \\
& =W(z) \exp \left(-c(z-p)^{N+1}\right) .
\end{aligned}
$$

We expand near $z=p$, using $W(p)=1$,

$$
\begin{aligned}
\Sigma(z) & =\left(1+W^{\prime}(p)(z-p)+\cdots+\frac{1}{(N+1) !} \frac{d^{N+1} W}{d z^{N+1}}(p)(z-p)^{N+1}+\cdots\right) \\
& \times\left(1-c(z-p)^{N+1}+\frac{c^{2}}{2}(z-p)^{2 N+2}+\cdots\right),
\end{aligned}
$$

so that our choice of $c=c_{\mu, Q, a}=\frac{1}{(N+1) !} \frac{d^{N+1} W}{d z^{N+1}}(p)$ guarantees that the Taylor expansion of $\Sigma(z)$ does not contain a term of order $(z-p)^{N+1}$, say

$$
\Sigma(z)=\sum_{j=0}^{\infty} b_{j}(z-p)^{j}
$$

with $b_{N+1}=0$. Thus we make sense of the whole R.H.S. in expression (32) as

$$
\begin{aligned}
- & (N+1)(z-p)^{N+1} \int \frac{\Sigma(z)}{(z-p)^{N+2}} \\
= & -(N+1)(z-p)^{N+1} \sum_{j=0}^{N} b_{j} \int \frac{d z}{(z-p)^{N+2-j}} \\
- & (N+1)(z-p)^{N+1} \int \frac{d z}{(z-p)^{N+2}}\left(\Sigma(z)-\sum_{j=0}^{N+1} b_{j}(z-p)^{j}\right) \\
= & \sum_{j=0}^{N} b_{j} \frac{N+1}{N+1-j}(z-p)^{j} \\
& -(N+1)(z-p)^{N+1} \int_{p}^{z} \frac{d w}{(w-p)^{N+2}}\left(\Sigma(w)-\sum_{j=0}^{N+1} b_{j}(w-p)^{j}\right) .
\end{aligned}
$$

The latter integral is well-defined since its argument function is holomorphic and $\Omega$ is simply connected. Let us note that for $b_{N+1} \neq 0$, an additional term would arise of the form $-(N+1) b_{N+1}(z-p)^{N+1} \ln (z-p)$ making the R.H.S. in (32) a multi-valued function.

Since $b_{0}=1$, let us stress that any solution $Q$ of (32) automatically satisfies $Q(p)=1$ and $Q-\frac{z-p}{N+1} Q^{\prime} \neq 0$ in $\Omega$, as required. For $a=0$ and $\varepsilon=0$, the constant $c_{\mu, Q, a}$ reduces to

$$
c_{0}=\frac{1}{(N+1) !} \frac{d^{N+1}}{d z^{N+1}}\left(e^{4 \pi(N+1) h_{p}(z)+k(z)}\right)(p),
$$


where $h_{p}(z)$ is the holomorphic extension of $H(z, p)-H(p, p)$ so that $h_{p}(p)=0$. Correspondingly, (25) has the solution $Q_{0}$ given by:

$Q_{0}(z)=-(N+1)(z-p)^{N+1} \int \frac{d z}{(z-p)^{N+2}} \exp \left(4 \pi(N+1) h_{p}(z)+k(z)-c_{0}(z-p)^{N+1}\right)$.

By the Implicit Function Theorem, we have the following existence result.

Lemma 3.1. For a and $\varepsilon>0$ small, in a small neighborhood of $Q_{0}$ there exists a unique holomorphic function $Q_{\mu, a} \in C(\bar{\Omega}, \mathbb{C})$ (depending smoothly on $\mu$ and a) satisfying (32):

$$
\begin{aligned}
Q(z)=-(N+1)(z-p)^{N+1} \int \frac{d z}{(z-p)^{N+2}} \exp \begin{array}{r}
\left(4 \pi h_{Q, a}(z)+r_{\mu, Q, a}(z)\right. \\
\left.+k(z)-c_{\mu, Q, a}(z-p)^{N+1}\right) .
\end{array}
\end{aligned}
$$

Proof. Let $H=\{Q \in C(\bar{\Omega}, \mathbb{C}): Q$ is holomorphic in $\Omega\}$. For given $\mu>0$, define the map

$$
\begin{aligned}
P: \mathbb{R}^{+} \times H \times \mathbb{C} & \rightarrow H \\
(\varepsilon, Q, a) & \rightarrow Q(z)+(N+1)(z-p)^{N+1} \int \frac{\Sigma(z)}{(z-p)^{N+2}},
\end{aligned}
$$

where $\Sigma(z)=\exp \left(4 \pi h_{Q, a}(z)+r_{\mu, Q, a}(z)+k(z)-c_{\mu, Q, a}(z-p)^{N+1}\right)$. We have that

$$
\begin{aligned}
& P(0, Q, 0)=Q+(N+1)(z-p)^{N+1} \int \frac{1}{(z-p)^{N+2}} \exp \left(4 \pi(N+1) h_{p}(z)\right. \\
& \left.+k(z)-c_{0}(z-p)^{N+1}\right) .
\end{aligned}
$$

Since $\partial_{Q} P\left(0, Q_{0}, 0\right)=\operatorname{Id}$ and $P\left(0, Q_{0}, 0\right)=0$, by the Implicit Function Theorem we find $\varepsilon_{0}>0$ small and a smooth map $(\varepsilon, a) \in\left(0, \varepsilon_{0}\right) \times B_{\varepsilon_{0}}(0) \rightarrow Q_{\varepsilon, \mu, a} \in H$ so that $Q_{0, \mu, 0}=Q_{0}$ and $P\left(\varepsilon, Q_{\varepsilon, \mu, a}, a\right)=0$. The required smallness of $\varepsilon_{0}$ is easily shown to be independent of $\mu$, and $Q_{\varepsilon, \mu, a}$ depends smoothly also on $\mu$, provided $\mu$ remains bounded and bounded away from zero. To keep light notation, we will omit the explicit dependence of $Q_{\varepsilon, \mu, a}$ on $\varepsilon$ and simply write $Q_{\mu, a}$.

Next, for $a=0$ and $\varepsilon=0$, equation (26) has a unique solution

$$
\mu_{0}=\frac{e^{4 \pi(N+1) H(p, p)+K(p)}}{\sqrt{8}(N+1)} .
$$

By perturbation, we get

Lemma 3.2. For $a$ and $\varepsilon>0$ small, in a small neighborhood of $\mu_{0}$ there exists a unique solution $\mu_{a}$ (depending smoothly on a) to (26).

Proof. Set

$$
\begin{aligned}
T: \mathbb{R}^{+} \times \mathbb{R} \times \mathbb{C} & \rightarrow \mathbb{R} \\
(\varepsilon, \mu, a) & \rightarrow \mu-\frac{e^{4 \pi H_{Q_{\mu, a}, a}(p)+R_{\mu, Q_{\mu, a}, a}(p)+K(p)}}{\sqrt{8}(N+1)} .
\end{aligned}
$$

Since $Q_{\mu, a}$ depends smoothly on $\mu$ and $a$, the same regularity holds for $T$. We have that

$$
T(0, \mu, 0)=\mu-\frac{e^{4 \pi(N+1) H(p, p)+K(p)}}{\sqrt{8}(N+1)} .
$$

Since $T\left(0, \mu_{0}, 0\right)=0$ and $\partial_{\mu} T\left(0, \mu_{0}, 0\right)=1$, we find $\varepsilon_{0}>0$ small and a smooth map

$$
(\varepsilon, a) \in\left(0, \varepsilon_{0}\right) \times B_{\varepsilon_{0}}(0) \mapsto \mu_{\varepsilon, a}
$$


so that $\mu_{0,0}=\mu_{0}$ and $T\left(\varepsilon, \mu_{\varepsilon, a}, a\right)=0$, by means of the Implicit Function Theorem. As before, we simply write $\mu_{a}$ instead of $\mu_{\varepsilon, a}$, and the proof is complete.

\section{Proofs of the main Results}

Proof of Theorem 2.1. Let $\Lambda \Subset \Omega$ be a region as in the statement of the theorem and set

$$
\Psi(p):=\frac{d^{N+1}}{d z^{N+1}}\left(e^{4 \pi(N+1) h_{p}(z)+k(z)}\right)(p) .
$$

Finding a solution of Problem (20) as desired reduces then to finding $p \in \Lambda$ such that

$$
\Psi_{a, \varepsilon}(p)=\frac{d^{N+1}}{d z^{N+1}}\left(e^{4 \pi h_{Q_{a}, a}(z)+r_{\mu_{a}, Q_{a}, a}(z)+k(z)}\right)(p)=0,
$$

where $Q_{a}=Q_{\mu_{a}, a}$, with $Q_{\mu, a}$ and $\mu_{a}$ given by Lemmas 3.1 and 3.2 , respectively. Since $\Psi_{a, \varepsilon}(p)$ depends continuously on $a, \varepsilon, p$ and $\Psi_{0,0}(p)=\Psi(p)$, we get that the degree of $\Psi_{a, \varepsilon}(p)$ on $\Lambda$ w.r.t. 0 is well-defined, for $|a|+\varepsilon$ small, and coincides with $\operatorname{deg}(\Psi, \Lambda, 0)$. The latter one being nontrivial by assumption, for $a$ and $\varepsilon>0$ small we find a solution $p_{a, \varepsilon} \in \Lambda$ of $\Psi_{a, \varepsilon}(p)=0$.

In this way, the function

$$
v_{\varepsilon}=\ln \frac{8(N+1)^{2} \mu_{a}^{2}\left|Q_{a}(z)-\frac{z-p}{N+1} Q_{a}^{\prime}(z)\right|^{2}}{\left(\mu_{a}^{2} \varepsilon^{2}\left|Q_{a}(z)\right|^{2}+\left|\left(z-p_{a, \varepsilon}\right)^{N+1}-a Q_{a}(z)\right|^{2}\right)^{2}}
$$

is a solution to Problem (20) with $p=p_{a, \varepsilon}$ for which the concentration property (28) easily follows. Since $\Psi_{a, \varepsilon}\left(p_{a, \varepsilon}\right)=0$, we finally have that $\Psi\left(p_{a, \varepsilon}\right) \rightarrow 0$ as $|a|+\varepsilon \rightarrow 0$.

Proof of Theorem 1.1. To establish Theorem 1.1, we observe that in the homogeneous case $\varphi=0, K(z)=-2 \pi N H(z, p)$ and the function $\Psi$ becomes

$$
\Psi(p)=\frac{d^{N+1}}{d z^{N+1}}\left(e^{2 \pi(N+2) h_{p}(z)}\right)(p) .
$$

We will compute the total degree of this map by estimating its behavior near $\partial \Omega$. Let us observe that

$$
H(z, p)-\frac{1}{2 \pi} \ln |z-\hat{p}| \rightarrow 0 \quad \text { in } C^{N+1}(\bar{\Omega}) \text { as } p \rightarrow \partial \Omega,
$$

where $\hat{p} \in \mathbb{R}^{2} \backslash \bar{\Omega}$ is the reflection of $p$ with respect to $\partial \Omega$. Let us stress that the reflection map is well-defined for points in $\Omega$ that are near $\partial \Omega$. The $C^{0}$-validity of (33) follows from the Maximum Principle applied to the harmonic function $H(z, p)-$ $\frac{1}{2 \pi} \ln |z-\hat{p}|$ by means of the asymptotic behavior

$$
H(z, p)-\frac{1}{2 \pi} \ln |z-\hat{p}|=\frac{1}{2 \pi} \ln \frac{|z-p|}{|z-\hat{p}|} \rightarrow 0 \text { unif. on } \partial \Omega, \text { as } p \rightarrow \partial \Omega .
$$

Elliptic regularity (see [17]) then implies the validity of (33).

Let us denote $d=\operatorname{dist}(p, \partial \Omega)$. Then, from (33), we obtain

$$
\begin{aligned}
& H(p, p)=\frac{1}{2 \pi} \ln |p-\hat{p}|+o(1)=\frac{1}{2 \pi} \ln (2 d)+o(1), \\
& H(z, p)-H(p, p)=\frac{1}{2 \pi} \ln \frac{|z-\hat{p}|}{2 d}+o(1) \quad \text { in } C^{N+1}(\bar{\Omega})
\end{aligned}
$$


as $d \rightarrow 0$. We extend $H(z, p)-H(p, p)$ holomorphically in $\Omega$ by $h_{p}(z)$ with $h_{p}(p)=$ 0 , and as $d \rightarrow 0$, the expansion

$$
h_{p}(z)=\frac{1}{2 \pi} \ln \frac{z-\hat{p}}{2 d}+o(1) \quad \text { in } C^{N+1}(\bar{\Omega})
$$

holds. Since as $d \rightarrow 0$,

$$
e^{2 \pi(N+2) h_{p}(z)}=\left(\frac{z-\hat{p}}{2 d}\right)^{N+2}(1+o(1))
$$

in $C^{N+1}(\bar{\Omega})$, we finally get that in the homogeneous case,

$$
\Psi(p)=(N+2) ! \frac{p-\hat{p}}{2 d}(1+o(1))
$$

as $d \rightarrow 0$. But $\frac{p-\hat{p}}{2 d}=\nu_{\Omega}(p+\hat{p} / 2)$, where $\nu_{\Omega}(x)$ is the inward unit normal vector at $x \in \partial \Omega$ and $p+\hat{p} / 2$ is the projection of $p$ onto the boundary. The winding number of $\nu_{\Omega}$ along $\partial \Omega$ is \pm 1 and, by stability, we get that

$$
\operatorname{deg}\left(\Psi, 0, \Omega_{\delta}\right)= \pm 1 \neq 0
$$

for $\delta>0$ small. Here, $\Omega_{\delta}=\{x \in \Omega: d>\delta\}$.

Theorem 2.1 thus applies with $\Lambda=\Omega_{\delta}$ to provide, for $a$ and $\varepsilon>0$ small, $p_{a, \varepsilon} \in \Omega_{\delta}$ and solutions $v_{\varepsilon}$ to Problem (20) with $p=p_{a, \varepsilon}$ for which the concentration property (28) holds. Setting $\psi_{\varepsilon}(z)=v_{\varepsilon}(z)-4 \pi N G(z, p)$, the function $\psi_{\varepsilon}$ is a solution to Problem (5) with $\alpha=N, p=p_{a, \varepsilon}$ and (28) rewritten in terms of $\omega_{\varepsilon}$ as (18). The proof is concluded.

\section{ACKNOWLEDGEMENTS}

The work for this paper began while the second author was visiting the Departamento de Matematica, Pontificia Universidad Catolica de Chile (Santiago, Chile). He would like to thank M. Musso and M. del Pino for their kind invitation and hospitality.

\section{REFERENCES}

[1] S. Baraket, F. Pacard, Construction of singular limits for a semilinear elliptic equation in dimension 2. Calc. Var. Partial Differential Equations 6 (1998), no. 1, 1-38. MR.1488492 (98j:35057)

[2] D. Bartolucci, C.-C. Chen, C.-S. Lin, G. Tarantello, Profile of blow-up solutions to mean field equations with singular data. Comm. Partial Differential Equations 29 (2004), no. 7-8, 1241-1265. MR2097983 (2005i:58022)

[3] D. Bartolucci, G. Tarantello, Liouville type equations with singular data and their applications to periodic multivortices for the electroweak theory. Comm. Math. Phys. 229 (2002), no. 1, 3-47. MR1917672 (2003e:58026)

[4] H. Brezis, F. Merle, Uniform estimates and blow-up behavior for solutions of $-\Delta u=V(x) e^{u}$ in two dimensions. Comm. Partial Differential Equations 16 (1991), no. 8-9, 1223-1253. MR:1132783 (92m:35084)

[5] E. Caglioti, P.L. Lions, C. Marchioro, M. Pulvirenti, A special class of stationary flows for two-dimensional Euler equations: A statistical mechanics description. Comm. Math. Phys. 143 (1992), 501-525. MR.1145596 (93i:82060)

[6] E. Caglioti, P.L. Lions, C. Marchioro, M. Pulvirenti, A special class of stationary flows for two-dimensional Euler equations: A statistical mechanics description Part II. Comm. Math. Phys. 174 (1995), 229-260. MR1362165 (96k:82059)

[7] C.-C. Chen, C.-S. Lin, Sharp estimates for solutions of multi-bubbles in compact Riemann surfaces. Comm. Pure Appl. Math. 55 (2002), no. 6, 728-771. MR.1885666 (2003d:53056)

[8] C.-C. Chen, C.-S. Lin, Topological degree for a mean field equation on Riemann surfaces. Comm. Pure Appl. Math. 56 (2003), no. 12, 1667-1727. MR2001443(2004h:35065) 
[9] W. Chen, C. Li, Classification of solutions of some nonlinear elliptic equations. Duke Math. J. 63 (1991), 615-623. MR1121147 (93e:35009)

[10] K.S. Chou, T.Y.H. Wan, Asymptotic radial symmetry for solutions of $\Delta u+e^{u}$ in a punctured disc. Pacific J. of Math. 163 (1994), 269-276. MR.1262297 (95a:35038)

[11] M. Crandall, P.H. Rabinowitz, Some continuation and variational methods for positive solutions of nonlinear elliptic eigenvalue problems. Arch. Rational Mech. Anal. 58 (1975), no. 3, 207-218. MR0382848 (52:3730)

[12] M. del Pino, M. Kowalczyk, M. Musso, Singular limits in Liouville-type equations. Calc. Var. Partial Differential Equations 24 (2005), no. 1, 47-81. MR2157850 (2006h:35089)

[13] P. Esposito, Blow up solutions for a Liouville equation with singular data. Recent advances in elliptic and parabolic problems (Taiwan, 2004), 39-57, edited by Chiun-Chuan Chen, Michel Chipot and Chang-Shou Lin. MR2172566 (2006e:35098)

[14] P. Esposito, Blow up solutions for a Liouville equation with singular data. SIAM J. Math. Anal. 36 (2005), no. 4, 1310-1345. MR2139452 (2005m:35088)

[15] P. Esposito, M. Grossi, A. Pistoia, On the existence of blowing-up solutions for a mean field equation. Ann. Inst. Henri Poincaré Analyse Non Linéaire 22, no. 2 (2005), 227-257. MR2124164 (2005k:35112)

[16] I.M. Gelfand, Some problems in the theory of quasilinear equations. Amer. Math. Soc. Transl. 29 (1963), no. 2, 295-381. MR0153960(27:3921)

[17] D. Gilbarg, N.S. Trudinger, Elliptic partial differential equations of second order. 2nd Edition, Springer-Verlag, 1983. MR737190 (86c:35035)

[18] J. Han, J. Jang, Self-dual Chern-Simons vortices on bounded domains. Lett. Math. Phys. 64 (2003), 45-56. MR1997718(2004e:58020)

[19] D.D. Joseph, T.S. Lundgren, Quasilinear problems driven by positive sources. Arch. Rat. Mech. Anal. 49 (1973), 241-269. MR0340701(49:5452)

[20] M.H.K. Kiessling, Statistical mechanics of classical particles with logarithmic interaction. Comm. Pure Appl. Math. 46 (1993), 27-56. MR1193342 (93k:82003)

[21] Y.-Y. Li, Harnack type inequality: The method of moving planes. Comm. Math. Phys. 200 (1999), 421-444. MR.1673972 (2000c:58024)

[22] Y.-Y. Li, I. Shafrir, Blow-up analysis for solutions of $-\Delta u=V e^{u}$ in dimension two. Indiana Univ. Math. J. 43 (1994), no. 4, 1255-1270. MR1322618 (96c:35048)

[23] P.-L. Lions, Mathematical topics in fluid mechanics. Vol. 1. Incompressible models. Oxford Lecture Series in Mathematics and its Applications, 3. Oxford Science Publications. The Clarendon Press, Oxford University Press, New York, 1996. MR1422251 (98b:76001)

[24] J. Liouville, Sur l'Equation aux Difference Partielles $\frac{d^{2} \log \lambda}{d u d v} \pm \frac{\lambda}{2 a^{2}}=0$. C.R. Acad. Sci. Paris 36 (1853), 71-72.

[25] A. Majda, A. Bertozzi, Vorticity and incompressible flow. Cambridge Texts in Applied Mathematics, 27. Cambridge University Press, Cambridge, 2002. MR.1867882 (2003a:76002)

[26] L. Ma, J. Wei, Convergence for a Liouville equation. Comment. Math. Helv. 76 (2001), 506-514. MR.1854696 (2002h:35097)

[27] K. Nagasaki, T. Suzuki, Asymptotic analysis for two-dimensional elliptic eigenvalue problems with exponentially dominated nonlinearities. Asymptotic Anal. 3 (1990), no. 2, 173-188. MR:1061665 (91f:35053)

[28] M. Nolasco, G. Tarantello, Double vortex condensates in the Chern-Simons-Higgs theory. Calc. Var. Partial Differential Equations 9 (1999), 31-91. MR1710938 (2001e:58022)

[29] J. Prajapat, G. Tarantello, On a class of elliptic problem in $\mathbb{R}^{2}$ : Symmetry and uniqueness results. Proc. Roy. Soc. Edinburgh Sect. A 131 (2001), 967-985. MR1855007 (2002j:35108)

[30] J.T. Stuart, On finite amplitude oscillations in laminar mixing layers. J. Fluid. Mech. 29 (1967), no. 3, 417-440.

[31] T. Suzuki, Two-dimensional Emden-Fowler equation with exponential nonlinearity. Nonlinear diffusion equations and their equilibrium states, 3 (Gregynog, 1989), 493-512. Progr. Nonlinear Differential Equations Appl., 7, Birkhäuser Boston, Boston, MA, 1992. MR1167859 (93e:35041)

[32] G. Tarantello, Multiple condensate solutions for the Chern-Simons-Higgs theory. J. Math. Phys. 37 (1996), 3769-3796. MR1400816 (97f:58045)

[33] G. Tarantello, Analytical aspects of Liouville-type equations with singular sources. Stationary partial differential equations. Vol. I, 491-592, Handbook Differ. Equ., North-Holland, Amsterdam, 2004. MR 2103693(2006e:35124) 
[34] G. Tarantello, A quantization property for blow up solutions of singular Liouville-type equations. J. Funct. Anal. 219 (2005), no. 2, 368-399. MR2109257(2005i:35073)

[35] A. Tur, V. Yanovsky, Point vortices with a rational necklace: New exact stationary solutions of the two-dimensional Euler equation. Phys. Fluids 16 (2004), no. 8, 2877-2885. MR 2075552 (2005b:76029)

[36] V.H. Weston, On the asymptotic solution of a partial differential equation with an exponential nonlinearity. SIAM J. Math. Anal. 9 (1978), no. 6, 1030-1053. MR512508 (80a:35022)

Departamento de Ingeniería Matemática and CMM, Universidad de Chile, Casilla 170, Correo 3, Santiago, Chile

E-mail address: delpino@dim.uchile.cl

Dipartimento di Matematica, Università degli Studi "Roma Tre", Largo S. Leonardo Murialdo, 1, 00146 Roma, Italy

E-mail address: esposito@mat.uniroma3.it

Dipartimento di Matematica, Politecnico di Torino, Corso Duca Degli Abruzzi, 24, 10129 Torino, Italy - and - Departamento de Matematica, Pontificia Universidad Catolica de Chile, Avenida Vicuna Mackenna 4860, Macul, Santiago, Chile

E-mail address: mmusso@mat.puc.cl 